\title{
Dopamine (3-Hydroxytyramine) Metabolism in Parkinsonism
}

\author{
McC. Goodall and Harold Alton \\ From the Department of Surgery and Physiology, University of Texas Medical \\ Branch, Galveston, Texas 77550
}

\begin{abstract}
A в S T RACT Three patients with idiopathic parkinsonism and six normal subjects were infused over a $4 \mathrm{hr}$ period with $104.6 \mu \mathrm{c}$ of dopamine-2- ${ }^{14} \mathrm{C}$ (3,4-dihydroxyphenylethylamine, 3-hydroxytyramine), ${ }^{1}$ the immediate precursor in the synthesis of the sympathetic neurohormone, noradrenaline (norepinephrine). Urine was collected during the infusion period, $0-2 \mathrm{hr}, 2-4 \mathrm{hr}$, 4-8 hr, 8-24 hr, and thereafter for 4 additional days. Using a technique herein described, the various metabolic and biosynthetic products of dopamine, including noradrenaline and its metabolic products, were separated, identified, and their radioactivity measured.

The metabolic pattern of dopamine in the normal subject was compared to that of the three parkinsonism patients. The results indicate that in idiopathic parkinsonism there is a decrease in the recovery of free radioactive noradrenaline in the urine following an infusion of dopamine-2 ${ }^{14} \mathrm{C}$ and a slight shift toward dopamine metabolism. The latter is reflected by an increase in the following metabolites of dopamine : 3,4-dihydroxyphenylacetic acid and the conjugates of 3-methoxy-4-hydroxyphenylacetic acid, 3,4-dihydroxyphenylacetic acid, 3methoxy-4-hydroxyphenylethanol and dopamine.
\end{abstract}

\section{INTRODUCTION}

Dopamine (3,4-dihydroxyphenylethylamine, 3-hydroxytyramine) was originally synthesized in 1910 by Mannich and Jacobsohn (1). However, it was not until 1950 that this amine was found in mammalian tissue, at which time Goodall demonstrated its presence in the heart $(2,3)$ and in the adrenal gland along with L-DOPA (3,4-dihydroxyphenylalanine) $(3,4)$. Since then dopamine has been demonstrated in multiple tissues (5-12), urine (13-16), and brain $(17,18)$. In 1959, Bertler and Rosengren (19), Sano et al. (20), and later others (2123) showed that dopamine existed in relatively high

\footnotetext{
${ }^{1}$ Dopamine-2-14 $\mathrm{C} \quad \mathrm{HCl}$ (3,4-dihydroxyphenylethylamine, 3-hydroxytyramine), Nuclear-Chicago Corporation, Des Plaines, Ill.

Received for publication 14 February 1969 and in revised form 25 July 1969.
}

concentrations in the corpus striatum. However, it was Ehringer and Hornykiewicz (24) who first reported a decreased dopamine in the corpus striatum of patients with parkinsonism; subsequently, others confirmed this finding (25-27). Barbeau, Murphy, and Sourkes demonstrated a decreased urinary output of dopamine in parkinsonism (28). More recently L-DOPA has been used in the treatment of parkinsonism with varied success (29$38)$; nevertheless, Cotzias et al. $(37,38)$ showed that with large daily doses (from $300 \mathrm{mg}$ to $8 \mathrm{~g}$ ) of L-DOPA a sustained improvement could be produced.

It is well established that L-DOPA and dopamine are precursors to noradrenaline, (norepinephrine) (39-45). Further, it has been shown that the sympathetic nerves and ganglia synthesize noradrenaline, but not adrenaline (42); noradrenaline is also found in the higher centers $(46-50)$ including the basal ganglia $(20-24,26)$. Recent evidence seems to indicate that dopamine is a neurohormone with transmitter functions in the central nervous system $(22,26,27,51-65)$. In view of these findings and the fact that both dopamine and noradrenaline are decreased in the caudate, putamen, and substantia nigra in parkinsonism (24), attention is naturally focused on the interrelationship of these two amines in this disease, and more specifically, as to whether or not there is an aberration in synthesis or metabolism of dopamine and/or noradrenaline in parkinsonism. Therefore, these experiments were designed to evaluate more precisely the metabolic and precursor product relationship of dopamine in parkinsonism.

\section{METHODS}

General. Dopamine metabolism and noradrenaline synthesis were evaluated in three patients with idiopathic parkinsonism and six normal healthy male subjects between 21 and $35 \mathrm{yr}$. The dopamine metabolism and the noradrenaline synthesis of the two groups were compared. All medication was discontinued on the parkinsonism patients $3-4$ wk before the dopamine infusion. The patients as well as the normal subjects were maintained on bed rest during the infusion and for $24 \mathrm{hr}$ postinfusion. Thereafter, patients and subjects alike were maintained in sedentary states during the remaining collection periods. Each patient was selected in order 


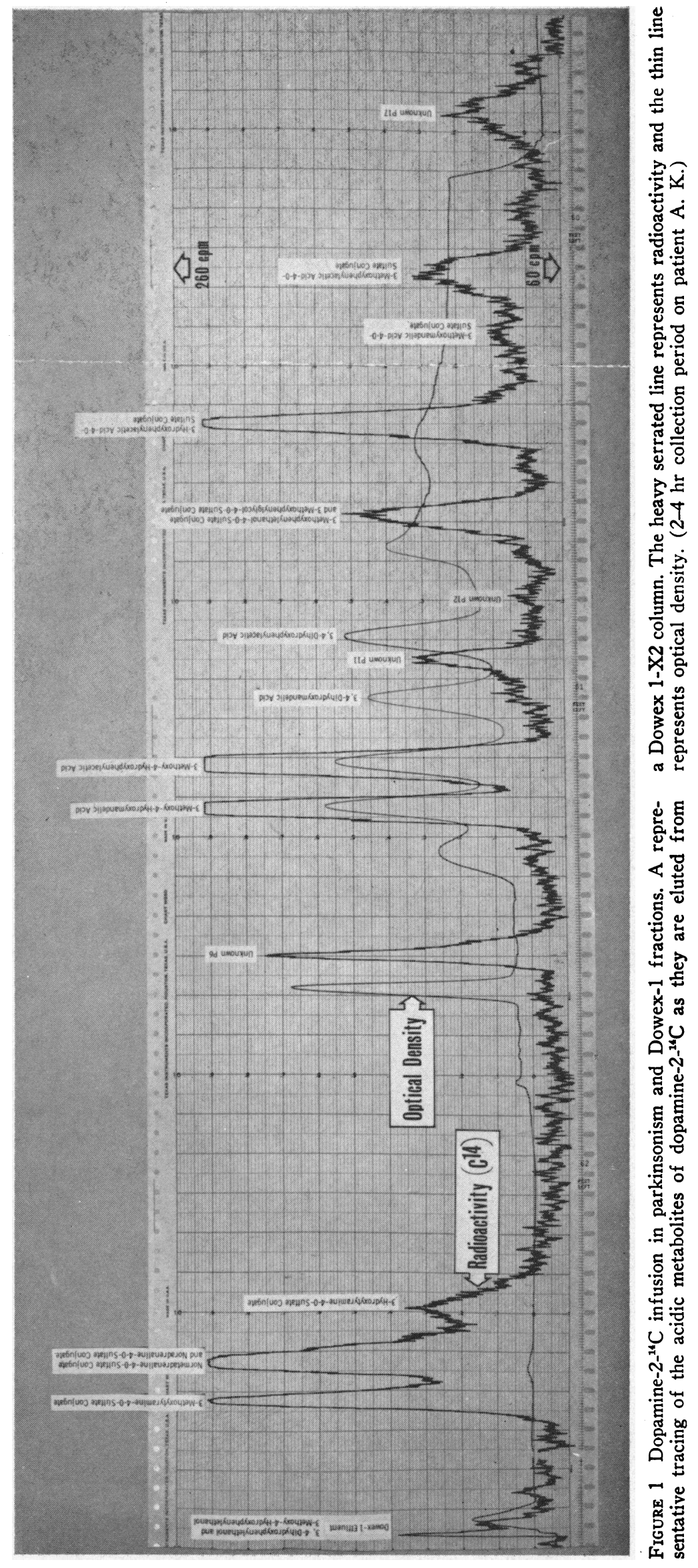


TABLE I

Excretion Pattern of the Basic Metabolites, Acidic Metabolites, and Biosynthetic Products of Dopamine after a 4 hr

\begin{tabular}{|c|c|c|c|c|c|c|c|c|c|c|c|c|c|}
\hline \multirow{2}{*}{$\begin{array}{l}\text { Period of urine } \\
\text { collection }\end{array}$} & \multirow{2}{*}{ Subject } & \multirow{2}{*}{$\begin{array}{l}\% \text { of the } \\
\text { infused } \\
\text { dose }\end{array}$} & \multicolumn{7}{|c|}{$\%$ of radioactivity recovered in each collection period } & \multicolumn{4}{|c|}{ 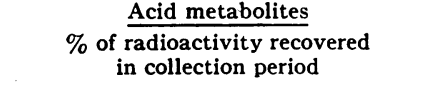 } \\
\hline & & & Adr & Noradr & Metadr & NM & $3-\mathrm{HT}$ & 3-MT & Unk & $\begin{array}{l}\text { Dowex-1 } \\
\text { effuent }\end{array}$ & $P_{2}$ Unk & $\begin{array}{l}\text { 3-MT } \\
\text { conj }\end{array}$ & $\begin{array}{l}\text { NM } \\
\text { conj }\end{array}$ \\
\hline \multirow[t]{2}{*}{ During infusion } & $\begin{array}{l}\text { A. K. } \\
\text { C. J. } \\
\text { B. G. }\end{array}$ & $\begin{array}{l}39.4 \\
40.7 \\
33.7\end{array}$ & $\begin{array}{l}\operatorname{Tr} \\
\operatorname{Tr} \\
\operatorname{Tr}\end{array}$ & $\begin{array}{l}\operatorname{Tr} \\
\operatorname{Tr} \\
\operatorname{Tr}\end{array}$ & $\begin{array}{l}\operatorname{Tr} \\
\operatorname{Tr} \\
\operatorname{Tr}\end{array}$ & $\begin{array}{l}\operatorname{Tr} \\
0.2 \\
\operatorname{Tr}\end{array}$ & $\begin{array}{r}1.1 \\
7.5 \\
13.8\end{array}$ & $\begin{array}{l}\operatorname{Tr} \\
\operatorname{Tr} \\
\operatorname{Tr}\end{array}$ & $\begin{array}{l}1.5 \\
\operatorname{Tr} \\
\operatorname{Tr}\end{array}$ & $\begin{array}{l}3.8 \\
1.3 \\
\operatorname{Tr}\end{array}$ & $\begin{array}{l}\operatorname{Tr} \\
0.6 \\
1.6\end{array}$ & $\begin{array}{l}3.5 \\
5.6 \\
4.0\end{array}$ & $\begin{array}{l}2.1 \\
6.6 \\
4.1\end{array}$ \\
\hline & Normal & $38.9 \pm 3.6$ & $\operatorname{Tr}$ & $2.9 \pm 0.3$ & $\mathrm{Tr}$ & $0.2 \pm 0.1$ & $8.1 \pm 1.5$ & $\operatorname{Tr}$ & $1.1 \pm 0.7$ & $1.7 \pm 0.2$ & $1.0 \pm 0.4$ & $3.6 \pm 1.1$ & $2.8 \pm 0.7$ \\
\hline \multirow[t]{2}{*}{$2 \mathrm{hr}$ postinfusion } & $\begin{array}{l}\text { A. K. } \\
\text { C. J. } \\
\text { B. G. }\end{array}$ & $\begin{array}{l}17.3 \\
16.8 \\
17.3\end{array}$ & $\begin{array}{l}\operatorname{Tr} \\
\operatorname{Tr} \\
\operatorname{Tr}\end{array}$ & $\begin{array}{l}\text { Tr } \\
0.3 \\
0.3\end{array}$ & $\begin{array}{l}\operatorname{Tr} \\
\operatorname{Tr} \\
\operatorname{Tr}\end{array}$ & $\begin{array}{l}\operatorname{Tr} \\
0.1 \\
0.2\end{array}$ & $\begin{array}{l}0.3 \\
0.1 \\
1.9\end{array}$ & $\begin{array}{l}\operatorname{Tr} \\
\operatorname{Tr} \\
\operatorname{Tr}\end{array}$ & $\begin{array}{l}0.4 \\
0.2 \\
0.2\end{array}$ & $\begin{array}{l}1.3 \\
0.7 \\
\operatorname{Tr}\end{array}$ & $\begin{array}{l}0.8 \\
0.2 \\
0.9\end{array}$ & $\begin{array}{r}7.4 \\
10.9 \\
5.5\end{array}$ & $\begin{array}{r}5.7 \\
11.4 \\
6.3\end{array}$ \\
\hline & Normal & $16.5 \pm 2.1$ & $\operatorname{Tr}$ & $0.9 \pm 0.3$ & $\operatorname{Tr}$ & $0.3 \pm 0.1$ & $0.9 \pm 0.4$ & $\operatorname{Tr}$ & $0.2 \pm 0.1$ & $1.0 \pm 0.2$ & $0.5 \pm 0.3$ & $8.0 \pm 2.3$ & $6.3 \pm 0.1$ \\
\hline \multirow[t]{2}{*}{$2-4 \mathrm{hr}$} & $\begin{array}{l}\text { A. K. } \\
\text { C. J. } \\
\text { B. G. }\end{array}$ & $\begin{array}{l}8.4 \\
9.4 \\
9.9\end{array}$ & $\begin{array}{l}\operatorname{Tr} \\
\operatorname{Tr} \\
\operatorname{Tr}\end{array}$ & $\begin{array}{l}0.5 \\
0.3 \\
0.1\end{array}$ & $\begin{array}{l}\operatorname{Tr} \\
\operatorname{Tr} \\
\operatorname{Tr}\end{array}$ & $\begin{array}{l}0.3 \\
0.3 \\
0.5\end{array}$ & $\begin{array}{l}0.2 \\
\mathrm{Tr} \\
0.1\end{array}$ & $\begin{array}{l}\operatorname{Tr} \\
\operatorname{Tr} \\
\operatorname{Tr}\end{array}$ & $\begin{array}{l}\operatorname{Tr} \\
0.3 \\
\operatorname{Tr}\end{array}$ & $\begin{array}{l}0.6 \\
0.9 \\
0.6\end{array}$ & $\begin{array}{l}0.6 \\
0.2 \\
0.5\end{array}$ & $\begin{array}{r}10.7 \\
14.3 \\
8.6\end{array}$ & $\begin{array}{l}10.4 \\
16.4 \\
11.1\end{array}$ \\
\hline & Normal & $9.6 \pm 1.5$ & $\operatorname{Tr}$ & $0.6 \pm 0.2$ & $\operatorname{Tr}$ & $0.3 \pm 0.1$ & $0.2 \pm 0.1$ & $\operatorname{Tr}$ & $\operatorname{Tr}$ & $0.9 \pm 0.2$ & $0.4 \pm 0.2$ & $10.4 \pm 1.4$ & $7.8 \pm 0.7$ \\
\hline $4-8 \mathrm{hr}$ & $\begin{array}{l}\text { A. K. } \\
\text { C. J. } \\
\text { B. G. }\end{array}$ & $\begin{array}{l}11.8 \\
10.2 \\
11.5\end{array}$ & $\begin{array}{l}\mathrm{Tr} \\
\mathrm{Tr} \\
\mathrm{Tr}\end{array}$ & $\begin{array}{l}0.1 \\
0.5 \\
0.4\end{array}$ & $\begin{array}{l}\mathrm{Tr} \\
\mathrm{Tr} \\
\mathrm{Tr}\end{array}$ & $\begin{array}{l}0.2 \\
0.3 \\
0.2\end{array}$ & $\begin{array}{l}0.4 \\
\mathrm{Tr} \\
0.2\end{array}$ & $\begin{array}{l}\mathrm{Tr} \\
\mathrm{Tr} \\
\mathrm{Tr}\end{array}$ & $\begin{array}{l}0.1 \\
1.0 \\
\text { Tr }\end{array}$ & $\begin{array}{l}1.2 \\
1.1 \\
0.7\end{array}$ & $\begin{array}{l}0.8 \\
\operatorname{Tr} \\
0.7\end{array}$ & $\begin{array}{r}9.1 \\
16.5 \\
10.4\end{array}$ & $\begin{array}{r}6.5 \\
11.0 \\
8.4\end{array}$ \\
\hline \multirow{3}{*}{$8-24 \mathrm{hr}$} & Normal & $9.1 \pm 0.5$ & $\mathrm{Tr}$ & $0.7 \pm 0.1$ & $\operatorname{Tr}$ & $0.4 \pm 0.1$ & $0.2 \pm 0.1$ & $\operatorname{Tr}$ & $\operatorname{Tr}$ & $0.8 \pm 0.3$ & $0.6 \pm 0.2$ & $14.3 \pm 2.0$ & $8.4 \pm 1.8$ \\
\hline & $\begin{array}{l}\text { A. K. } \\
\text { C. J. } \\
\text { B. G. }\end{array}$ & $\begin{array}{l}16.1 \\
15.6 \\
16.3\end{array}$ & $\begin{array}{l}\mathrm{Tr} \\
\mathrm{Tr} \\
\mathrm{Tr}\end{array}$ & $\begin{array}{l}0.2 \\
0.2 \\
0.4\end{array}$ & $\begin{array}{l}\mathrm{Tr} \\
\mathrm{Tr} \\
\mathrm{Tr}\end{array}$ & $\begin{array}{l}0.2 \\
0.3 \\
0.3\end{array}$ & $\begin{array}{l}0.3 \\
\mathrm{Tr} \\
0.3\end{array}$ & $\begin{array}{l}\operatorname{Tr} \\
\operatorname{Tr} \\
\operatorname{Tr}\end{array}$ & $\begin{array}{l}\mathrm{Tr} \\
0.2 \\
\mathrm{Tr}\end{array}$ & $\begin{array}{l}0.8 \\
1.0 \\
1.0\end{array}$ & $\begin{array}{l}0.9 \\
0.6 \\
0.9\end{array}$ & $\begin{array}{r}9.9 \\
14.1 \\
8.3\end{array}$ & $\begin{array}{r}8.2 \\
5.1 \\
10.9\end{array}$ \\
\hline & Normal & $13.5 \pm 2.1$ & $\operatorname{Tr}$ & $0.7 \pm 0.1$ & $\operatorname{Tr}$ & $0.3 \pm 0.1$ & $0.1 \pm 0.1$ & $\operatorname{Tr}$ & $\operatorname{Tr}$ & $0.8 \pm 0.3$ & $0.9 \pm 0.3$ & $9.5 \pm 2.1$ & $5.5 \pm 1.6$ \\
\hline \multirow[t]{2}{*}{$24-48 \mathrm{hr}$} & $\begin{array}{l}\text { A. K. } \\
\text { C. J. } \\
\text { B. G. }\end{array}$ & $\begin{array}{l}2.2 \\
5.4 \\
6.0\end{array}$ & $\begin{array}{l}\mathrm{Tr} \\
\mathrm{Tr} \\
\mathrm{Tr}\end{array}$ & $\begin{array}{l}\mathrm{Tr} \\
0.1 \\
0.4\end{array}$ & $\begin{array}{l}\mathrm{Tr} \\
\mathrm{Tr} \\
\mathrm{Tr}\end{array}$ & $\begin{array}{l}\operatorname{Tr} \\
0.2 \\
0.1\end{array}$ & $\begin{array}{l}\mathrm{Tr} \\
\mathrm{Tr} \\
0.2\end{array}$ & $\begin{array}{l}\mathrm{Tr} \\
\mathrm{Tr} \\
\mathrm{Tr}\end{array}$ & & $\begin{array}{l}0.8 \\
1.0 \\
0.8\end{array}$ & $\begin{array}{l}\mathrm{Tr} \\
1.2 \\
0.8\end{array}$ & $\begin{array}{r}7.7 \\
13.2 \\
4.6\end{array}$ & $\begin{array}{l}5.4 \\
3.8 \\
4.6\end{array}$ \\
\hline & Normal & $5.6 \pm 1.0$ & $\operatorname{Tr}$ & $0.5 \pm 0.1$ & $\operatorname{Tr}$ & $0.5 \pm 0.1$ & & $\mathrm{Tr}$ & & $0.7 \pm 0.2$ & $1.0 \pm 0.3$ & $6.1 \pm 2.1$ & $4.6 \pm 0.1$ \\
\hline \multirow[t]{2}{*}{$48-72 \mathrm{hr}$} & $\begin{array}{l}\text { A. K. } \\
\text { C. J. } \\
\text { B. G. }\end{array}$ & $\begin{array}{l}1.7 \\
1.7 \\
3.2\end{array}$ & $\begin{array}{l}\operatorname{Tr} \\
\operatorname{Tr} \\
\operatorname{Tr}\end{array}$ & $\begin{array}{l}\mathrm{Tr} \\
\mathrm{Tr} \\
\mathbf{0 . 5}\end{array}$ & $\begin{array}{l}\mathrm{Tr} \\
\mathrm{Tr} \\
\mathrm{Tr}\end{array}$ & $\begin{array}{l}\operatorname{Tr} \\
\operatorname{Tr} \\
0.3\end{array}$ & & $\begin{array}{l}\mathrm{Tr} \\
\mathrm{Tr} \\
\mathrm{Tr}\end{array}$ & & $\begin{array}{l}1.3 \\
\operatorname{Tr} \\
0.9\end{array}$ & $\begin{array}{l}0.8 \\
0.7 \\
0.9\end{array}$ & $\begin{array}{l}7.1 \\
6.0 \\
2.0\end{array}$ & $\begin{array}{l}5.4 \\
5.1 \\
5.1\end{array}$ \\
\hline & Normal & $2.2 \pm 0.4$ & $\operatorname{Tr}$ & $0.5 \pm 0.1$ & $\operatorname{Tr}$ & $0.5 \pm 0.1$ & & $\operatorname{Tr}$ & & $1.3 \pm 0.5$ & $1.0 \pm 0.3$ & $3.2 \pm 0.7$ & $3.9 \pm 1.0$ \\
\hline \multirow[t]{2}{*}{$72-96 \mathrm{hr}$} & $\begin{array}{l}\text { A. K. } \\
\text { C. J. } \\
\text { B. G. }\end{array}$ & $\begin{array}{l}0.6 \\
0.6 \\
1.3\end{array}$ & $\begin{array}{l}\mathrm{Tr} \\
\mathrm{Tr} \\
\mathrm{Tr}\end{array}$ & & $\begin{array}{l}\mathrm{Tr} \\
\operatorname{Tr} \\
\mathrm{Tr}\end{array}$ & & & $\begin{array}{l}\operatorname{Tr} \\
\operatorname{Tr} \\
\operatorname{Tr}\end{array}$ & & $\begin{array}{l}2.6 \\
4.8 \\
4.4\end{array}$ & $\begin{array}{l}\mathrm{Tr} \\
\mathrm{Tr} \\
\mathrm{Tr}\end{array}$ & $\begin{array}{l}6.2 \\
7.0 \\
1.5\end{array}$ & $\begin{array}{l}2.4 \\
6.2 \\
3.1\end{array}$ \\
\hline & Normal & $1.2 \pm 0.3$ & $\operatorname{Tr}$ & & $\operatorname{Tr}$ & & & $\operatorname{Tr}$ & & $2.3 \pm 1.0$ & $1.0 \pm 0.5$ & $2.5 \pm 0.4$ & $3.7 \pm 0.4$ \\
\hline \multirow[t]{3}{*}{$96-120 \mathrm{hr}$} & $\begin{array}{l}\text { A. K. } \\
\text { C. J. } \\
\text { B. G. }\end{array}$ & $\begin{array}{l}0.3 \\
\operatorname{Tr} \\
0.6\end{array}$ & $\begin{array}{l}\mathrm{Tr} \\
\mathrm{Tr} \\
\mathrm{Tr}\end{array}$ & & $\begin{array}{l}\mathrm{Tr} \\
\mathrm{Tr} \\
\mathrm{Tr}\end{array}$ & & & $\begin{array}{l}\operatorname{Tr} \\
\operatorname{Tr} \\
\operatorname{Tr}\end{array}$ & & 3.8 & 1.1 & $\operatorname{Tr}$ & 7.7 \\
\hline & Normal & $0.6 \pm 0.2$ & $\operatorname{Tr}$ & & $\operatorname{Tr}$ & & & $\operatorname{Tr}$ & & $2.8 \pm 1.0$ & $\operatorname{Tr}$ & $2.6 \pm 1.0$ & $3.8 \pm 1.1$ \\
\hline & & & \multicolumn{7}{|c|}{$\%$ of infused dose } & \multicolumn{4}{|c|}{$\%$ of infused dose } \\
\hline $\begin{array}{l}\text { Beginning infusion to } \\
24 \mathrm{hr}, \% \text { of dose } \\
\text { recovered }\end{array}$ & $\begin{array}{l}\text { A. } \mathbf{K} \text {. } \\
\text { C. J. } \\
\text { B. G. }\end{array}$ & $\begin{array}{l}93.0 \\
92.7 \\
88.7\end{array}$ & $\begin{array}{l}\operatorname{Tr} \\
\operatorname{Tr} \\
\operatorname{Tr}\end{array}$ & $\begin{array}{l}0.1 \\
0.6 \\
0.2\end{array}$ & $\begin{array}{l}\operatorname{Tr} \\
\operatorname{Tr} \\
\operatorname{Tr}\end{array}$ & $\begin{array}{l}0.1 \\
0.2 \\
0.1\end{array}$ & $\begin{array}{l}0.6 \\
3.1 \\
5.1\end{array}$ & $\begin{array}{l}\operatorname{Tr} \\
\operatorname{Tr} \\
\operatorname{Tr}\end{array}$ & $\begin{array}{l}0.7 \\
0.2 \\
\text { Tr }\end{array}$ & $\begin{array}{l}2.0 \\
1.0 \\
0.3\end{array}$ & $\begin{array}{l}0.4 \\
0.4 \\
1.1\end{array}$ & $\begin{array}{l}6.2 \\
9.3 \\
5.7\end{array}$ & $\begin{array}{l}4.8 \\
8.1 \\
6.3\end{array}$ \\
\hline \multirow{3}{*}{ To $120 \mathrm{hr}$} & Normal & $87.6 \pm 3.1$ & $\operatorname{Tr}$ & $1.5 \pm 0.2$ & $\operatorname{Tr}$ & $0.2 \pm 0.1$ & $3.3 \pm 0.7$ & $\operatorname{Tr}$ & $0.4 \pm 0.3$ & $1.1 \pm 0.2$ & $0.7 \pm 0.3$ & $6.3 \pm 1.4$ & $4.5 \pm 0.7$ \\
\hline & $\begin{array}{l}\text { A. } \mathbf{K} . \\
\text { C. J. } \\
\text { B. G. }\end{array}$ & $\begin{array}{r}97.8 \\
100.4 \\
99.8\end{array}$ & $\begin{array}{l}\operatorname{Tr} \\
\operatorname{Tr} \\
\operatorname{Tr}\end{array}$ & $\begin{array}{l}0.1 \\
0.6 \\
0.2\end{array}$ & $\begin{array}{l}\operatorname{Tr} \\
\operatorname{Tr} \\
\operatorname{Tr}\end{array}$ & $\begin{array}{l}0.1 \\
0.2 \\
0.2\end{array}$ & $\begin{array}{l}0.6 \\
3.1 \\
5.1\end{array}$ & $\begin{array}{l}\operatorname{Tr} \\
\operatorname{Tr} \\
\operatorname{Tr}\end{array}$ & $\begin{array}{l}0.7 \\
0.2 \\
\text { Tr }\end{array}$ & $\begin{array}{l}2.1 \\
1.1 \\
0.5\end{array}$ & $\begin{array}{l}0.4 \\
0.5 \\
1.1\end{array}$ & $\begin{array}{r}6.5 \\
10.2 \\
6.1\end{array}$ & $\begin{array}{l}5.0 \\
8.4 \\
6.8\end{array}$ \\
\hline & Normal & $97.2 \pm 3.5$ & $\operatorname{Tr}$ & $1.5 \pm 0.2$ & $\operatorname{Tr}$ & $0.3 \pm 0.1$ & $3.3 \pm 0.7$ & $\operatorname{Tr}$ & $0.4 \pm 0.3$ & $1.2 \pm 0.2$ & $0.8 \pm 0.3$ & $6.7 \pm 1.5$ & $4.8 \pm 0.7$ \\
\hline
\end{tabular}

Adr, adrenaline: Noradr, noradrenaline; Metadr, metadrenaline; NM, normetadrenaline; 3-HT, 3-hydroxytyramine (dopamine) ; 3-MT, 3-methoxytyramine; Unk, unknown; Conj, conjugate; MOMA, 3-methoxy-4-hydroxymandelic acid; HVA, 3-methoxy-4-hydroxyphenylacetic acid; DOMA, 3,4-dihydroxymandelic acid; DOPAC, 3,4-dihydroxyphenylacetic acid; MHPG Conj, 3-methoxy-4-hydroxyphenylglycol conjugate; MHPE Conj, 3-methoxy-4hydroxyphenylethanol conjugate; $\mathrm{Tr}$, trace. 
Acid metabolites

$\%$ of radioactivity recovered in collection period

\begin{tabular}{|c|c|c|c|c|c|c|c|c|c|c|c|c|c|}
\hline $\begin{array}{l}\text { Noradr } \\
\text { conj }\end{array}$ & $\begin{array}{l}\text { 3-HT } \\
\text { conj }\end{array}$ & P。 Unk & MOMA & HVA & DOMA & $P_{11}$ Unk & DOPAC & $P_{12}$ Unk & $\begin{array}{c}\text { MHPG \& } \\
\text { MHPE } \\
\text { conj }\end{array}$ & $\begin{array}{c}\text { DOPAC } \\
\text { conj }\end{array}$ & $\underset{\text { conj }}{\text { MOMA }}$ & $\begin{array}{l}\text { HVA } \\
\text { conj }\end{array}$ & $P_{17}$ Unk \\
\hline 2.9 & 1.0 & 0.8 & 3.3 & 47.1 & 3.0 & $\mathrm{Tr}$ & 10.2 & $\mathrm{Tr}$ & 3.6 & 4.8 & $\operatorname{Tr}$ & 7.0 & 4.2 \\
\hline 1.0 & 2.6 & 1.2 & 2.9 & 44.9 & 1.4 & 0.9 & 12.7 & $\mathrm{Tr}$ & 2.5 & 4.0 & $\mathrm{Tr}$ & 5.1 & 3.2 \\
\hline 1.4 & 2.6 & 3.0 & 1.7 & 31.1 & 2.5 & $\mathrm{Tr}$ & 9.1 & $\operatorname{Tr}$ & 4.3 & 9.2 & $\operatorname{Tr}$ & 6.5 & 5.4 \\
\hline $1.8 \pm 0.5$ & $1.0 \pm 0.3$ & $1.9 \pm 0.7$ & $2.3 \pm 0.4$ & $47.8 \pm 2.9$ & $\operatorname{Tr}$ & $1.9 \pm 1.0$ & $2.0 \pm 1.2$ & $1.4 \pm 0.5$ & $3.0 \pm 1.2$ & $3.9 \pm 0.7$ & $1.4 \pm 0.4$ & $5.3 \pm 2.0$ & $4.3 \pm 1.0$ \\
\hline 4.9 & 3.0 & 2.9 & 7.5 & 36.3 & 3.7 & $\mathrm{Tr}$ & 4.1 & $\mathrm{Tr}$ & 3.4 & 7.4 & 1.1 & 7.6 & 4.8 \\
\hline 2.7 & 2.9 & 2.1 & 8.7 & 23.5 & 3.0 & 0.9 & $\mathrm{Tr}$ & 1.1 & 7.0 & 6.1 & 0.7 & 11.6 & 7.4 \\
\hline 3.2 & 4.0 & 3.2 & 5.2 & 26.8 & 2.5 & 2.7 & 2.5 & $\operatorname{Tr}$ & 6.5 & 11.5 & 3.4 & 8.4 & 4.8 \\
\hline $3.2 \pm 0.5$ & $2.5 \pm 0.5$ & $2.5 \pm 1.1$ & $6.5 \pm 1.0$ & $36.3 \pm 4.2$ & $1.5 \pm 0.5$ & $4.1 \pm 1.0$ & $4.0 \pm 0.6$ & $1.5 \pm 1.3$ & $2.8 \pm 1.0$ & $7.2 \pm 2.2$ & $2.4 \pm 1.0$ & $6.4 \pm 2.1$ & $6.0 \pm 1.5$ \\
\hline 4.5 & 3.4 & 5.3 & 13.6 & 15.0 & 5.2 & 0.9 & 1.6 & $\operatorname{Tr}$ & 8.0 & 12.5 & 1.2 & 6.5 & 5.4 \\
\hline 2.5 & 3.3 & 3.8 & 13.9 & 8.4 & $\mathrm{Tr}$ & 0.9 & $\mathrm{Tr}$ & 1.1 & 9.8 & 8.4 & 1.6 & 7.2 & 7.3 \\
\hline 1.9 & 3.5 & 3.8 & 6.4 & 10.8 & 1.3 & 2.4 & $\mathrm{Tr}$ & 1.6 & 5.9 & 10.1 & 3.6 & 16.2 & 5.0 \\
\hline $4.4 \pm 0.4$ & $1.7 \pm 0.5$ & $4.3 \pm 1.3$ & $11.8 \pm 0.8$ & $20.4 \pm 1.6$ & $1.6 \pm 0.4$ & $1.5 \pm 0.4$ & $4.4 \pm 1.0$ & $1.3 \pm 0.4$ & $5.8 \pm 1.5$ & $8.6 \pm 1.9$ & $3.8 \pm 0.9$ & $6.0 \pm 0.9$ & $5.0 \pm 2.0$ \\
\hline 9.0 & 3.7 & 6.3 & 15.1 & 8.7 & 1.6 & 1.6 & 1.6 & 0.5 & 8.1 & 12.3 & 2.5 & 4.3 & 6.0 \\
\hline 5.4 & 3.8 & 4.4 & 15.7 & 2.9 & 1.2 & 0.8 & $\mathrm{Tr}$ & $\mathrm{Tr}$ & 13.6 & 9.8 & 1.7 & 5.7 & 7.2 \\
\hline 3.4 & 3.3 & 7.0 & 19.1 & 8.6 & 1.3 & 1.0 & 2.6 & 1.2 & 7.6 & 12.0 & 2.9 & 4.0 & 5.1 \\
\hline $4.5 \pm 0.9$ & $2.8 \pm 0.3$ & $7.6 \pm 0.5$ & $15.8 \pm 1.5$ & $6.5 \pm 1.4$ & $1.2 \pm 0.2$ & $2.0 \pm 0.7$ & $1.7 \pm 0.5$ & $2.0 \pm 0.6$ & $7.1 \pm 0.9$ & $9.0 \pm 1.4$ & $3.7 \pm 1.0$ & $4.8 \pm 1.8$ & $6.6 \pm 2.0$ \\
\hline 6.4 & 2.3 & 9.9 & 22.9 & 2.9 & 3.7 & $\mathrm{Tr}$ & 1.6 & $\mathrm{Tr}$ & 13.5 & 14.6 & $\mathrm{Tr}$ & 3.8 & 3.7 \\
\hline 3.9 & 2.3 & 4.0 & 24.1 & $\operatorname{Tr}$ & $\operatorname{Tr}$ & 0.8 & $\mathrm{Tr}$ & $T_{r}$ & 17.2 & 10.2 & 1.8 & 3.0 & 5.1 \\
\hline 6.2 & 2.8 & 9.7 & 17.7 & 2.2 & 0.4 & $\operatorname{Tr}$ & $\operatorname{Tr}$ & 1.2 & 11.4 & 13.6 & 2.5 & 3.6 & 6.3 \\
\hline $3.2 \pm 0.9$ & $2.3 \pm 0.8$ & $12.0 \pm 1.0$ & $23.4 \pm 3.7$ & $2.7 \pm 0.9$ & $0.9 \pm 0.4$ & $2.1 \pm 0.4$ & $\operatorname{Tr}$ & $2.5 \pm 0.7$ & $10.4 \pm 1.6$ & $11.1 \pm 3.0$ & $3.4 \pm 0.3$ & $4.7 \pm 0.3$ & $3.8 \pm 1.1$ \\
\hline 1.6 & 2.3 & 12.2 & 33.8 & $\mathrm{Tr}$ & $\mathrm{Tr}$ & 4.2 & $\mathrm{Tr}$ & 2.6 & 15.3 & 14.5 & $\mathrm{Tr}$ & 2.1 & 3.1 \\
\hline 2.2 & 1.6 & 7.3 & 30.8 & $\mathrm{Tr}$ & $\mathrm{Tr}$ & 0.9 & $\mathrm{Tr}$ & 2.0 & 19.3 & 11.9 & 1.7 & 2.7 & 4.3 \\
\hline 3.2 & 3.3 & 11.5 & 24.5 & 2.5 & $\operatorname{Tr}$ & 2.3 & $\mathrm{Tr}$ & 3.1 & 13.4 & 11.9 & 5.2 & 4.5 & 3.1 \\
\hline $1.2 \pm 0.2$ & $\operatorname{Tr}$ & $15.1 \pm 3.0$ & $35.9 \pm 3.8$ & $1.8 \pm 0.4$ & $\mathrm{Tr}$ & $2.3 \pm 0.2$ & $\operatorname{Tr}$ & $2.6 \pm 1.0$ & $11.6 \pm 2.1$ & $8.3 \pm 1.2$ & $2.8 \pm 1.0$ & $4.3 \pm 1.8$ & $2.2 \pm 0.6$ \\
\hline 1.2 & $\operatorname{Tr}$ & 11.4 & 37.5 & $\operatorname{Tr}$ & $\operatorname{Tr}$ & 3.8 & $\mathrm{Tr}$ & $\operatorname{Tr}$ & 16.5 & 14.2 & $\operatorname{Tr}$ & $\operatorname{Tr}$ & $\operatorname{Tr}$ \\
\hline 1.2 & 2.1 & 8.7 & 35.2 & $\mathrm{Tr}$ & $\mathrm{Tr}$ & Tr & $\mathrm{Tr}$ & 2.1 & 19.8 & 12.8 & 2.6 & $\mathrm{Tr}$ & 3.7 \\
\hline 0.9 & 2.2 & 12.2 & 27.3 & 1.6 & $\mathrm{Tr}$ & 2.2 & $\mathrm{Tr}$ & 3.3 & 11.6 & 10.1 & 3.7 & 6.2 & $\mathrm{Tr}$ \\
\hline $\operatorname{Tr}$ & $\mathrm{Tr}$ & $17.7 \pm 2.2$ & $39.0 \pm 1.6$ & $\operatorname{Tr}$ & $\operatorname{Tr}$ & $2.6 \pm 0.5$ & $\operatorname{Tr}$ & $2.8 \pm 0.7$ & $11.7 \pm 1.3$ & $6.6 \pm 1.2$ & $3.3 \pm 1.0$ & $2.5 \pm 0.5$ & $\operatorname{Tr}$ \\
\hline 5.9 & $\operatorname{Tr}$ & 10.8 & 42.0 & $\operatorname{Tr}$ & $\operatorname{Tr}$ & 4.3 & $\operatorname{Tr}$ & $\mathrm{Tr}$ & 13.3 & 12.6 & $\operatorname{Tr}$ & $\operatorname{Tr}$ & $\operatorname{Tr}$ \\
\hline 1.1 & $\operatorname{Tr}$ & 5.2 & 33.4 & $\mathrm{Tr}$ & $\mathrm{Tr}$ & $\mathrm{Tr}$ & $\mathrm{Tr}$ & 2.1 & 18.9 & 12.5 & 4.8 & $\mathrm{Tr}$ & 3.8 \\
\hline 1.1 & 2.3 & 13.8 & 39.8 & $\mathrm{Tr}$ & $\mathrm{Tr}$ & 1.6 & $\mathrm{Tr}$ & 2.2 & 12.7 & 9.7 & 2.7 & 2.4 & 2.6 \\
\hline $\operatorname{Tr}$ & $\operatorname{Tr}$ & $17.2 \pm 3.0$ & $42.1 \pm 3.5$ & $\operatorname{Tr}$ & $\operatorname{Tr}$ & $2.6 \pm 0.5$ & $\operatorname{Tr}$ & $2.9 \pm 0.3$ & $12.8 \pm 3.4$ & $8.2 \pm 2.1$ & $3.0 \pm 1.8$ & $1.9 \pm 0.5$ & $\operatorname{Tr}$ \\
\hline & & & & & & & & & & & 1 & & \\
\hline 1.8 & $\operatorname{Tr}$ & 14.7 & 31.5 & $\operatorname{Tr}$ & $\mathrm{Tr}$ & $\operatorname{Tr}$ & $\operatorname{Tr}$ & 5.5 & 13.5 & 9.5 & 1.5 & 5.2 & 4.0 \\
\hline $\operatorname{Tr}$ & $\operatorname{Tr}$ & $20.1 \pm 4.0$ & $34.4 \pm 4.6$ & $\mathrm{Tr}$ & $\mathrm{Tr}$ & $3.0 \pm 0.8$ & $\operatorname{Tr}$ & $2.5 \pm 0.6$ & $13.6 \pm 4.5$ & $8.8 \pm 3.0$ & $2.5 \pm 1.1$ & $4.1 \pm 0.6$ & $\operatorname{Tr}$ \\
\hline \multicolumn{14}{|c|}{$\%$ of infused dose } \\
\hline 4.4 & 2.0 & 3.6 & 9.2 & 27.6 & 3.0 & 0.3 & 5.3 & 0.1 & 5.8 & 8.0 & 0.6 & 5.7 & 4.2 \\
\hline 2.2 & 2.6 & 2.3 & 9.3 & 23.3 & 1.2 & 0.8 & 5.2 & 0.3 & 7.2 & 6.0 & 0.7 & 5.7 & 4.7 \\
\hline 2.6 & 2.7 & 4.3 & 7.2 & 17.5 & 1.6 & 0.8 & 3.8 & 0.5 & 5.9 & 9.7 & 1.7 & 6.3 & 4.7 \\
\hline $2.4 \pm 0.6$ & $1.5 \pm 0.4$ & $3.9 \pm 0.7$ & $7.7 \pm 1.0$ & $27.5 \pm 2.2$ & $0.6 \pm 0.2$ & $2.0 \pm 0.7$ & $2.0 \pm 0.7$ & $1.4 \pm 0.6$ & $4.2 \pm 1.1$ & $5.8 \pm 1.3$ & $2.1 \pm 0.5$ & $4.8 \pm 0.1$ & $4.2 \pm 1.1$ \\
\hline 4.6 & 2.1 & 4.1 & 10.8 & 27.6 & 3.0 & 0.4 & 5.3 & 0.1 & 6.5 & 8.6 & 0.6 & 5.8 & 4.3 \\
\hline 2.4 & 2.7 & 2.8 & 11.8 & 23.3 & 1.2 & 0.9 & 5.2 & 0.4 & 8.7 & 7.0 & 0.9 & 5.8 & 5.1 \\
\hline 2.9 & 3.0 & 5.7 & 10.2 & 17.7 & 1.6 & 1.0 & 3.8 & 0.8 & 7.3 & 10.9 & 2.1 & 6.8 & 5.0 \\
\hline $2.6 \pm 0.6$ & $1.5 \pm 0.4$ & $5.4 \pm 1.0$ & $11.3 \pm 1.4$ & $27.6 \pm 2.2$ & $0.6 \pm 0.2$ & $2.3 \pm 0.7$ & $2.0 \pm 0.7$ & $1.7 \pm 0.7$ & $5.4 \pm 1.3$ & $6.6 \pm 1.5$ & $2.4 \pm 0.5$ & $5.1 \pm 0.1$ & $4.4 \pm 1.2$ \\
\hline
\end{tabular}




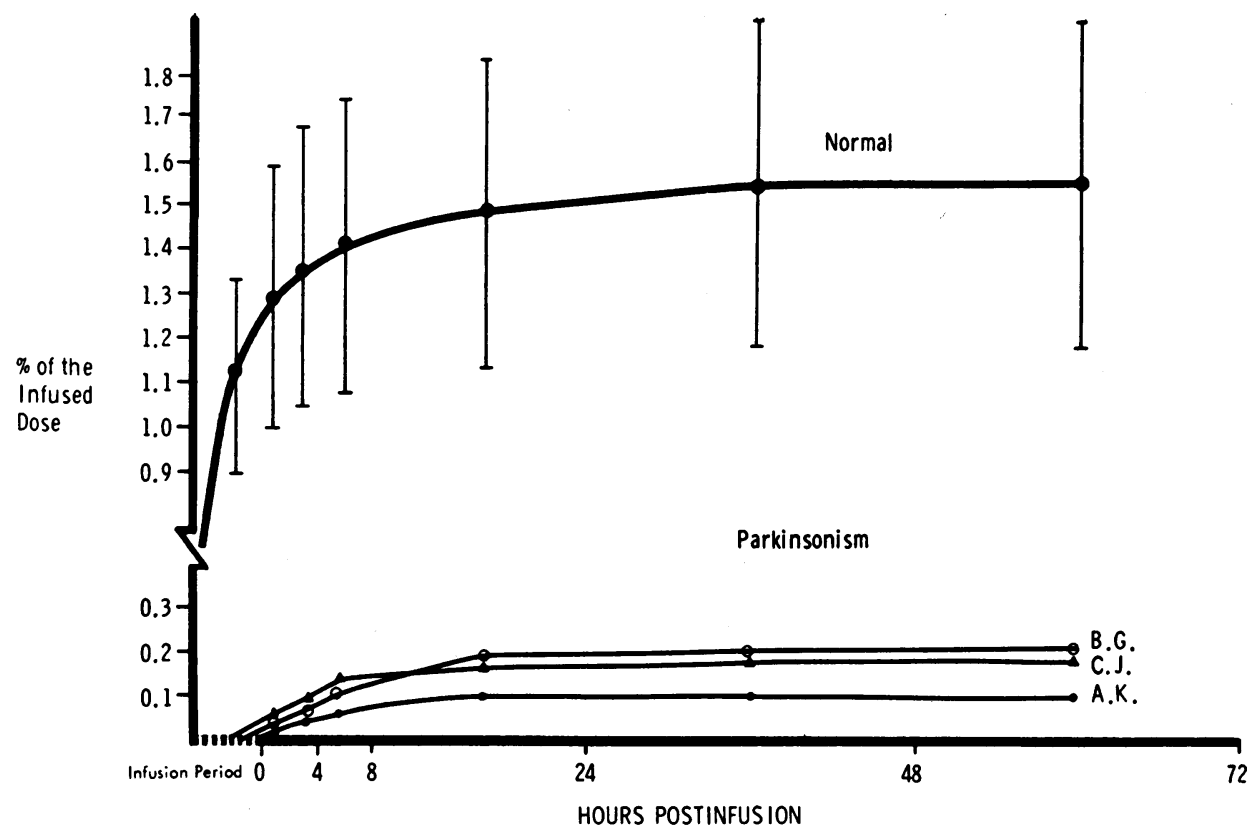

FIGURE 2 The accumulative recovery of the infused radioactivity as noradrenaline from the beginning of the infusion to $72 \mathrm{hr}$ after infusion of dopamine-2- ${ }^{14} \mathrm{C}$. A comparison between normal subjects and three patients with idiopathic parkinsonism.

to obtain typical cases of idiopathic parkinsonism, see case summaries as follows:

1. A. K, 57 yr old male. Onset 8 months before admission. Admission: BP 110/70, pulse 60 , resp. 20, temp. $98.6^{\circ}$. Past history: Noncontributory, no history of encephalitis or influenza. Laboratory: Blood and urine analysis within normal limits, normal chest X-ray, Grade I and II/IV systolic ejection murmur. Physical: Progressive weakness, masked facies, cogwheel rigidity in both arms, but in the right more than in the left, festinating gait, bilateral nonintention tremor of hands and right leg. No pathological reflexes. No sensory defects.

2. C. J., 58 yr old male. Estimated onset $25 \mathrm{yr}$ before admission but progressive for past $10 \mathrm{yr}$. Admission: $\mathrm{BP}$ $118 / 70$, pulse 72 , resp. 20 , temp. $98.0^{\circ}$. Past history: Noncontributory, no history of encephalitis or influenza. Laboratory: Blood and urine analysis within normal limits. Normal chest X-ray and electrocardiogram. Physical: Progressive weakness, impaired speech, masked facies, cogwheel rigidity, festinating gait, bilateral nonintention tremor of right and left hands, lip and jaw tremor. Deep tendon reflexes, equal bilaterally. Cranial nerves and sensation intact.

3. B. G., $69 \mathrm{yr}$ old male, onset $5 \mathrm{yr}$ before admission. Admission: BP $100 / 70$, pulse 72 , resp. 18 , temp. $98.6^{\circ}$. Past history: Noncontributory, no history of encephalitis or influenza, mother died of influenza, 1917. Laboratory: Blood and urine analysis within normal limits, except positive for venereal disease (VDRL), normal chest X-ray and electrocardiogram, negative brain scan. Physical: Progressive weakness, masked facies, cogwheel rigidity, festinating gait, bilateral nonintention tremor of left and right hands. The neurologic exam was otherwise normal except for mild impairment of vibratory sensation and light touch in extremities.
Each patient and normal subjects were infused with $104.8 \mu \mathrm{c}(872 \mu \mathrm{g})$ of dopamine-2 ${ }^{14} \mathrm{C}$, (3,4-dihydroxyphenylethylamine, 3-hydroxytyramine). The labeled dopamine was dissolved in $1000 \mathrm{ml}$ of physiological saline and infused via the antecubital vein over a $4 \mathrm{hr}$ period at a constant rate.

Urine was collected during the infusion period and at 2-hr intervals for the first $4 \mathrm{hr}$ postinfusion. It was then collected at the end of $8,24,48,72,96$, and $120 \mathrm{hr}$. The urine samples were frozen immediately and stored at $-20^{\circ} \mathrm{C}$ until assayed.

The method for isolating and quantifying endogenous noradrenaline and the metabolic products of noradrenaline, as well as the metabolic products of dopamine, has been previously described $(65,66)$. In brief, the basic metabolic products, separated on an Amberlite IRP- $64^{2}$ column, are adrenaline (epinephrine), metadrenaline (3-O-methyladrenaline, metanephrine), noradrenaline (norepinephrine), normetadrenaline (3-O-methylnoradrenaline, normetanephrine $\mathrm{NM}$ ), dopamine, and 3-methoxytyramine (3-MT).

The acidic metabolic products, separated on a column of Dowex-1-X $2^{3}$ acetate anion exchange resin, are 3-methoxy4-hydroxymandelic acid (MOMA), 3,4-dihydroxymandelic acid (DOMA), 3-methoxy-4-hydroxyphenylacetic acid (homovanillic acid, HVA), 3,4-dihydoxyphenylacetic acid (DOPAC), 3-methoxy-4-hydroxyphenylethanol (MHPE), 3,4-dihydoxyphenylethanol, 3-methoxy-4-hydroxy-phenylglycol (MHPG), 3,4-dihydroxyphenylglycol, vanillic acid (VA), and their respective conjugates as well as the conjugates of 3-methoxytyramine, normetadrenaline, noradrenaline, and dopamine. The details of these procedures have been described in a previous paper (76). Fig. 1 represents a typi-

Amberlite IRP-64, Rohm and Haas Co., Philadelphia, Pa. Dowex 1-X2, 200-400 mesh, Bio-Rad Laboratories, Richmond, Calif. 
cal elution pattern obtained in the separation of the acidic metabolic products. All acidic and basic metabolites were compiled and compared to those of normal subjects similarly infused with dopamine-2- ${ }^{14} \mathrm{C}$ (Table I).

\section{RESULTS}

The metabolic pattern of dopamine-2- ${ }^{14} \mathrm{C}$ in six normal subjects was compared to that of three patients with parkinsonism. Standard deviations were calculated on all metabolic products in the normal subjects but not in the parkinsonism patients since these patients very in degree from one another (Table I).

From the results it appears that the most important change occurred in the formation of noradrenaline. There was a significant decrease in the radioactivity recovered as noradrenaline throughout the collection periods (Table I and Fig. 2). As for the metabolic products of noradrenaline, there appeared to be a significant increase in DOMA during the infusion and early collection periods and a small but variable increase in NM conjugate (Table I). There was no significant change in the other metabolic products of noradrenaline, i.e., MOMA, normetadrenaline, vanillic acid, and the conjugate of 3-methoxy-4-hydroxyphenylglycol (MHPG).

As for the metabolic products of dopamine-2- ${ }^{14} \mathrm{C}$, there was a slight shift towards dopamine metabolism. This is reflected by an increase in the following metabolites of dopamine: DOPAC and the conjugates of HVA, DOPAC, 3-methoxy-4-hydroxyphenylethanol, and dopamine (Table I). There were no significant changes in 3-methoxytyramine or its conjugate. HVA was normal in two patients and decreased in patient B. G. However, it should be noted that HVA takes its origin from DOPAC, and therefore, any decrease in HVA could be reflected by an increase in DOPAC, DOPAC conjugate, or HVA conjugate as was the case with patient B. G.

Peaks 11 and 12 showed significant decreases in all patients, but since these are unknown compounds, very little can be said about them except that they represent a small percentage of the total radioactivity recovered in each collection period. Preliminary evidence indicates that these unknowns are metabolic products of dopamine rather than noradrenaline.

Approximately $75 \%$ of the infused dopamine was directly metabolized into dopamine-related metabolic products, i.e., 3-methoxytyramine, DOPAC, HVA, 3-methoxy-4-hydroxyphenylethanol (MHPE), 3,4-dihydroxyphenylethanol, and their respective conjugates, the conjugate of dopamine, and several unknowns (Table I). Of these metabolic products, HVA represents the largest single fraction (65). The remaining $25 \%$ of the infused dopamine- $2-{ }^{14} \mathrm{C}$ was synthesized into noradrenaline and appeared in the urine as radioactive noradrenaline or the metabolic products of noradrenaline which are DOMA, NM, NOMA, vanillic acid, and their 4-O sulfate conjugates, conjugates of 3-methoxy-4-hydroxyphenylglycol, and 3,4-dihydroxyphenylglycol (65, 67-69). Other secondary metabolites of noradrenaline such as HVA, DOPAC, and their 4-O sulfate conjugates are also present $(70,71)$, but in these experiments their origin is principally from dopamine.

Whereas all the parkinsonism patients showed the same general metabolic pattern following an infusion of dopamine-2- ${ }^{14} \mathrm{C}$, they, as to be expected, varied in degree one from another (Table I and Fig. 2).

\section{DISCUSSION}

It is well established that noradrenaline is the neurohormone of the sympathetic nerves $(42,46,72)$. Furthermore, noradrenaline is present in various parts of the brain $(46,50)$, including the corpus striatum $(20-24$, 26 ), but especially great is its concentration in the hypothalamus $(20,21,24,26)$. The immediate precursor to

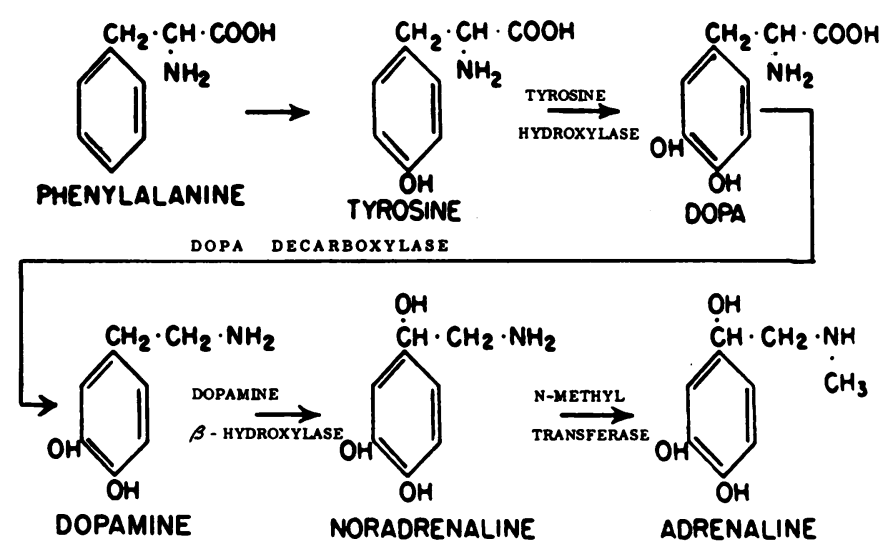

FIGURE 3 Biosynthetic pathway to the formation of noradrenaline (norepinephrine) and adrenaline (epinephrine). 
noradrenaline is dopamine and that to dopamine is DOPA (42) (Fig. 3). Recently, attention has been focused on dopamine as a neurohormone with transmitter functions in the central nervous system $(22,26,27$, 51-65), and of special significance is the high concentration of dopamine in the nucleus caudate, putamen, pallidum, and substantia nigra (19-27). Further, a decreased concentration of dopamine in the corpus striatum has been noted in parkinsonism (24-27). Whereas the introduction of dopamine might seem to be the logical approach to treating the depletion in idiopathic parkinsonism, nevertheless, this has not proven successful since the corpus striatum is considerably less permeable to circulating dopamine than it is to DOPA. In the corpus striatum the DOPA is readily decarboxylated to form dopamine. Therefore, efforts have been directed towards using the dopamine precursor, i.e. L-DOPA $(4,42)$ which in small amounts produced varied results (29-38) but in large daily doses produced a sustained improvement $(37,38)$.

The results of these experiments indicate that in patients with idiopathic parkinsonism there is a slight shift toward dopamine metabolism which is reflected by an increase in DOPAC and other dopamine metabolites (Table I). The increase in DOPAC observed principally in the infusion period has also been noted in patients with neurogenic orthostatic hypotension (66), but the meaning behind this increase remains unexplained. Nevertheless, this finding may be important since it could indicate a more local biochemical aberration of dopamine metabolism. Certainly the finding of decreased 3-methoxy-4-hydroxyphenylacetic acid (the principal metabolite of DOPAC) in the corpus striatum of parkinsonism patients (73) lends support to such a concept. Furthermore, since in parkinsonism the corpus striatum shows a deficit of dopamine (24-27), the immediate precursor to noradrenaline (42), one would then expect to also find a decrease of noradrenaline in these same structures and this too has been demonstrated $(26,74)$.

The question naturally arises as to whether or not the decrease in recovery of free radioactive noradrenaline in parkinsonism was related to debility, old age, or muscular inactivity. It is known that muscular exercise increases the release of noradrenaline (75) and this is reflected by an increase of noradrenaline in the urine. However, in these experiments the controls were maintained on bed rest during the infusion, for $24 \mathrm{hr}$ postinfusion, and thereafter in a sedentary state. Also, similar dopamine-2- ${ }^{14} \mathrm{C}$ infusion experiments were performed on three normal male subjects (20-25 yr) maintained on bed rest and were then repeated after these subjects exercised moderately. In these rest-exercise experiments, there was no significant change of free radioactive noradrenaline. Further, since old age and debility could effect the noradrenaline synthesis, similar experiments were performed on an elderly (62 yr), debilitated, bed ridden individual but the results indicated that in this patient the noradrenaline synthesis was within normal limits.

Results herein described indicate that in idiopathic parkinsonism there is a decrease in the recovery of free radioactive noradrenaline in the urine following an infusion of the immediate precursor to noradrenaline, i.e., dopamine-2- ${ }^{14} \mathrm{C}$ (Table I and Fig. 2). The pathogenesis of this diminished recovery of radioactive noradrenaline, renal function being normal, could be the result of an increase in noradrenaline metabolism or a decrease in its synthesis. Increased metabolism of noradrenaline is seen in the human during such stressful situations as severe thermal injury (76); however, in severe burns there is not only a decrease in the urinary recovery of radioactive noradrenaline but also a concomitant increase in noradrenaline metabolic products and a marked increase in the utilization rate of the infused dopamine $-2-{ }^{14} \mathrm{C}$ (76). In contrast, the opposite situation is seen in neurogenic hypotension where there is a decrease synthesis of noradrenaline with a concomitant decrease in noradrenaline metabolic products (66); and further, hypotension, a salient feature of neurogenic orthostatic hypotension, is sufficiently common to parkinsonism to have warranted speculation of a possible relationship between these two syndromes (7779). Therefore, this similarity with neurogenic hypotension and the decreased recovery of radioactive noradrenaline in idiopathic parkinsonism would seem to imply that in parkinsonism there is also an alteration in noradrenaline synthesis with a sympathetic nervous system involvement. However, the answer is considerably more complex than this indicates since in idiopathic parkinsonism there is also a decrease of dopamine in the corpus striatum (24-27) and the urine (28) and a decrease of HVA in the corpus striatum (73). This would clearly indicate that the decreased recovery of radioactive noradrenaline observed in these experiments could be secondary to a defect further back than dopamine in the synthesis of noradrenaline, i.e., in the conversion of DOPA to dopamine (DOPA is rapidly decarboxylated to dopamine) or tyrosine to DOPA or possibly phenylalanine to tyrosine.

\section{ACKNOWLEDGMENT}

This work was supported by U. S. Public Health Service Grant AM-12805.

\section{REFERENCES}

1. Mannich, C., and W. Jacobsohn. 1910. Uber Oxyphenylalkylamine und Dioxyphenyl-alkylamine. Ber. Deut. Chem. Ges. 1: 189.

2. Goodall, McC. 1950. Hydroxytyramine in mammalian heart. Nature (London). 166: 738. 
3. Goodall, McC. 1951. Studies of adrenaline and noradrenaline in mammalian heart and suprarenals. Acta Physiol. Scand. 24: Supp. 85.

4. Goodall, McC. 1950. Dihydroxyphenylalanine and hydroxytyramine in mammalian suprarenals. Acta Chem. Scand. 4: 550.

5. Schümann, H. J. 1956. Nachweis von Oxytyramin (Dopamin) in sympathischen Nerven und Ganglien. Arch. Pharmakol. Exp. Pathol. 227: 566.

6. von Euler, U. S., and F. Lishajko. 1957. Dopamine in mammalian lung and spleen. Acta Physiol. Pharmacol. Neer. 6: 295.

7. Dengler, H. 1957. Über das Vorkommen von Oxytyramin in der Nebenniere. Arch. Pharmakol. Exp. Pathol. $231: 373$.

8. von Euler, U. S., and F. Lishajko. 1958. Catechol amines in vascular wall. Acta Physiol. Scand. 42: 333.

9. Schümann, H. J. 1959. Über den Hydroxytyraminehalt der Organe. Arch. Pharmakol Exp. Pathol. 236: 474.

10. Angelakos, E. T., K. Fuxe, and M. L. Torchiana. 1963. Chemical and histochemical evaluation of the distribution of catecholamines in the rabbit and guinea pig hearts. Acta Physiol. Scand. 59: 184.

11. Bertler, A., B. Falck, C. G. Gottfries, L. Ljundgren, and E. Rosengren. 1964. Some observations on adrenergic connections between mesencephalon and cerebral hemispheres. Acta Pharmacol Toxicol. 21: 283.

12. Laverty, R., and D. F. Sharman. 1965. The estimation of small quantities of 3,4-dihydroxyphenylethylamine in tissues. Brit. J. Pharmacol. Chemother. 24: 538.

13. von Euler, U. S., U. Hamberg, and S. Hellner. 1951. B-(3,4-Dihydroxyphenyl) ethylamine (hydroxytyramine) in normal human urine. Biochem. J. 49: 655.

14. Drujan, B. D., T. L. Sourkes, D. S. Layne, and G. F. Murphy. 1959. The differential determination of catecholamines in urine. Can. J. Biochem. Physiol. 37: 1153.

15. Häggendal, J., and B. Werdinius. 1966. Dopamine in human urine during muscular work. Acta Physiol. Scand. 66: 223.

16. Holtz, P., K. Credner, and G. Kroneberg. 1947. Uber das sympothicomimetishe pressorische Priuzip des Harns ("Urosympotheir"). Arch. Pharmakol Exp. Pathol. 204: 228.

17. Montagu, K. A. 1957. Catechol compounds in rat tissues and in brains of different animals. Nature (London). $180: 244$.

18. Carlsson, A., M. Lindqvist, T. Magnusson, and B. Waldeck. 1958. On the presence of 3-hydroxytyramine in brain. Science. 127: 471.

19. Bertler, A., and E. Rosengren. 1959. Occurrence and distribution of dopamine in brain and other tissues. Experientia. 15 : 10.

20. Sano, I., T. Gamo, Y. Kakimoto, K. Taniguchi, M. Takesada, and K. Nishinuma. 1959. Distribution of catechol compounds in human brain. Biochim. Biophys. Acta. 32: 586.

21. Bertler, A., and E. Rosengren. 1959. Occurrence and distribution of catechol amines in brain. Acta Physiol. Scand. 47: 350 .

22. Anden, N. -E., A. Carlsson, A. Dahlstrom, K. Fuxe, N. -A. Hillarp, and K. Larsson. 1964. Demonstration and mapping out of nigro-neostriatal dopamine neurons. Life Sci. 3: 523.

23. Sourkes, T. L., and L. J. Poirier. 1966. Neurochemical basis of tremor and other disorders of movement. Can. Med. Ass. J. $94: 53$.
24. Ehringer, H., and O. Hornykiewicz. 1960. Verteilung von Noradrenalin und Dopamin (3-hydroxytyramin) im Gehirn des Menschen und ihr Verhalten bei Erkrankungen des Extrapyramidalen systems. Klin. Wochenschr. 38: 1236.

25. Sourkes, T. L. 1961. Formation of dopamine in vivo: relation to function of basal ganglia. Rev. Can. Biol. 20: 2.

26. Hornykiewicz, O. 1963. Die topische Lokalisation und das Verhalten von Noradrenalin und Dopamine (3-hydroxytyramin) in Parkinsonkranken Menschen. Wien. Klin. Wochenschr. 75: 309.

27. Carlsson, A. 1963. Evidence for a role of dopamine in extrapyramidal functions. Acta Neuroveget. (Vienna). 26: 484.

28. Barbeau, A., G. F. Murphy, and T. L. Sourkes. 1961. Excretion of dopamine in diseases of basal ganglia. Science. 133: 1706.

29. McGreer, P. L., and L. R. Zeldowicz. 1964. Administration of dihydroxyphenylalanine to Parkinsonian patients. Can. Med. Ass. J. 90: 463.

30. Fehling, C. 1966. Treatment of Parkinson's syndrome with L-DOPA a double blind study. Acta Neurol. Scand. 42: 367 .

31. Birkmayer, W., and O. Hornykiewicz. 1962. Der L-Dioxyphenylalanin (= L-DOPA). Effekt beim ParkinsonSyndrom des Menschen: zur Pathogenese und Behandlung der Parkinson-Akinese. Arch Psychiat. Nervenkr. 203: 560 .

32. Birkmayer, W., and O. Hornykiewicz. 1964. Weitere experimentelle Untersuchungen uber L-DOPA beim Parkinson-Syndrom und Reserpin-Parkinsonismus. Arch Psychiat. Nervenkr. 206: 367.

33. Barbeau, A., T. L. Sourkes, and G. F. Murphy. 1962. Les catecholamines dans la malade de Parkinson. In Monoamines et Systeme Nerveux Central. J. De Ajuriaguerra, editor. Masson et Cie, Paris. 247.

34. Gerstenbrand, F., K. Pateisky, and P. Prosenz. 1963. Erfahrungen mit L-DOPA in Therapie des Parkinsonismus. Psychiat. Neurol. 146: 246.

35. Friedhoff, A. F., L. Hekiman, M. Alpert, and E. Tobach. 1963. Dihydroxyphenylalanine in extrapyramididal disease. J. Amer. Med. Ass. 184: 285.

36. Bruno, A., and S. Cumer Bruno. 1966. Effects of L-DOPA on Pharmacological Parkinsonism. Acta Psychiat. Scand. $42: 64$.

37. Cotzias, G. C., M. H. Van Woert, and L. M. Schiffer. 1967. Aromatic amino acids and modification of Parkinsonism. N. Engl. J. Med. 276: 374.

38. Cotzias, G. C., P. S. Papavasilious, R. Gellene, R. B. Aronson, and I. Mena. 1969. Long term effects of DOPA on Parkinsonism: a proposal. Proc. 3rd Symposium on Parkinson's Disease. F. John Gillingham and I. M. L. Donaldson, editors. Livingstone Ltd., (E. \& S.). Edinburgh, Scotland. 178-181.

39. Blaschko, H. 1939. The specific action of L-DOPA decarboxylase. J. Physiol. (London). 13: 96.

40. Gurin, S., and A. M. Delluva. 1947. The biological synthesis of radioactive adrenaline from phenylalanine. $J$. Biol. Chem. 170: 545.

41. Goodall, McC., and N. Krishner. 1957. The biosynthesis of adrenaline and noradrenaline in vitro. J. Biol Chem. 266: 213.

42. Goodall, McC., and N. Kirshner. 1958. Biosynthesis of epinephrine and norepinephrine by sympathetic nerves and ganglia. Circulation. 17: 366 . 
43. Udenfriend, S., and J. B. Wyngarden. 1956. Precursors of adrenal epinephrine and norepinephrine in vivo. Biochim. Biophys. Acta. 20: 48.

44. Pellerin, J., and A. D'Iorio. 1957. Metabolism of DL-3,4dihydroxyphenylalanine-2-C14 in bovine adrenal homogenate. Can. J. Biochem. Physiol. 35: 151.

45. Spector, A., A. Sjoerdsma, P. Saltman-Nirenberg, M. Levitt, and S. Udenfriend. 1963. Norepinephrine synthesis from tyrosine-C14 in isolated perfused guinea pig hearts. Science. 139: 1299.

46. von Euler, U. S. 1955. Noradrenaline. Springfield, Illinois. Charles $\mathrm{C}$ Thomas, Publisher.

47. Merrit, J. H., and E. J. Schultz. 1966. The effect of decaborane on the biosynthesis and metabolism of norepinephrine in the rat brain. Life Sci. 5: 27.

48. Glowinski, J., and R. J. Baldessarini. 1966. Metabolism of norepinephrine in the central nervous system. Pharmacol. Rev. 18: 1201.

49. Rutledge, C. O., and J. Jonason. 1967. Metabolic pathways of dopamine and norepinephrine in rabbit brain in vitro. J. Pharmacol. Exp. Ther. 157: 493.

50. Vogt, M. 1954. The concentration of sympathin in different parts of the central nervous system under normal conditions and after administration of drugs. J. Physiol. (London). 123: 451.

51. Carlsson, A., M. Lindqvist, T. Magnusson, and B. Waldeck. 1958. On the presence of 3-hydroxytyramine in brain. Science. 127: 471.

52. Fuxe, K. 1965. Evidence for the Existence of Monoamine Neurons in the Central Nervous System. Almqvist \& Wiksells, Publishers, Stockholm.

53. Hornykiewicz, O. 1964. Zur Frage des Verlaufs dopaminerger Neurone im Gehirn des Menschen. Wien. Klin. Wochenschr. 76: 834.

54. Bertler, A. 1961. Occurrence and localization of catecholamines in the human brain. Acta Physiol. Scand. 51: 97.

55. Wiegand, R. G., and J. E. Perry. 1961. Effects of L-DOPA and $N$-methyl- $N$-benzyl-2-propyhylamine-HCl on DOPA, dopamine, norepinephrine, epinephrine and serotonin levels in mouse brain. Biochem. Pharmacol. 7 : 181.

56. Holtz, P., K. Stock, and E. Westermann. 1963. Uber die Blutdruckwirkung des Dopamins. Arch. Pharmakol Exp. Pathol. 246: 133

57. Fuxe, K., Th. Hökfelt, and O. Nilsson. 1964. Observations on the cellular localization of dopamine in the caudate nucleus of the rat. $Z$. Zellforsch. 63: 701 .

58. Häggendal, J., and T. Malmfors. 1963. Evidence of dopamine containing neurons in the retina of rabbits. Acta Physiol. Scand. 59: 295.

59. Bertler, A. 1964. Biochemical and Neurophysiological Correlations of Centrally Acting Drugs. Pergamon Press, Oxford.

60. Carlsson, A. 1966. Physiological and pharmacological release of monoamine in the central nervous system. In Mechanism of Release of Biogenic Amines, Proceedings of an International Wenner-Gren Symposium. Pergamon Press, Oxford. 331.

61. Poirier, L. J., and T. L. Sourkes. 1965. Influence of the substantia nigra on the catecholamine content of the striatum. Brain. 88: 181.
62. Heymans, C., and A. DeSchaepdryver. 1965. Dopamine and central neurotransmission. Pontif. Acad. Sci. Acta. 761.

63. Hornykiewicz, O. 1966. Dopamine (3-hydroxytyramine) and brain function. Pharmacol. Rev. 18: 925.

64. Austin, L., B. G. Livett, and I. W. Chubb. 1967. Increased synthesis and release of noradrenaline and dopamine during nerve stimulation. Life Sci. 6: 97.

65. Goodall, McC., and H. Alton. 1968. Metabolism of 3hydroxytyramine (dopamine) in human subjects. Biochem. Pharmacol. 17: 905.

66. Goodall, McC., W. R. Harlan, Jr., and H. Alton. 1968. Decreased noradrenaline (norepinephrine) synthesis in neurogenic orthostatic hypotension. Circulation. 38: 592.

67. Goodall, $\mathrm{McC}$., and H. Alton. 1969. Metabolism in the human of 3,4-dihydroxymandelic acid, one of the metabolites of noradrenaline and adrenaline. Biochem. Pharmacol. 18: 295.

68. Rosen, L., and McC. Goodall. 1962. Identification of vanillic acid as a catabolite of noradrenaline metabolism in the human. Proc. Soc. Exp. Biol. Med. 110: 767.

69. Goodall, McC., N. Kirshner, and L. Rosen. 1959. Metabolism of noradrenaline in the human. J. Clin. Invest. 38: 707.

70. Goodall, McC., and H. Alton. 1968. Preliminary identification of 3,4-dihydroxyphenylacetic acid and 3-methoxy4-hydroxyphenylacetic acid in human urine as metabolites of epinephrine. Tex. Rep. Biol. Med. 26: 107.

71. Alton, Harold, and McC. Goodall. 1969. Metabolism of 3,4-dihydroxyphenylacetic acid (DOPAC) in the human. Biochem. Pharmacol. 18: 1373.

72. von Euler, U. S. 1948. Identification of the sympathomimetic ergone in adrenergic nerves of cattle (sympathin N) with laevonoradrenaline. Acta Physiol. Scand. 16: 63 .

73. Bernheimer, H., and O. Hornykiewicz. 1965. Herabgesetzte Konzentration der Homovanillinsaure im $\mathrm{Ge}$ hirn von Parkinsonkranken Menschen als Ausdruck der Storung des Zentralen Dopaminstoffwechsels. Klin. Wochenschr. 43: 711.

74. Bernheimer, H., W. Birkmayer, and O. Hornykiewicz. 1963. Zur Biochemie des Parkinson-Syndroms des Menschen Einflu der Mono-aminoxydase-Hemmer-Therapic auf die Konzentration des Dopamins, Noradrenalins und 5-hydroxytryptamins im Gehirn. Klin. Wochenschr. 41: 465 .

75. von Euler, U. S. 1956. Noradrenaline: Chemistry, Physiology, Pharmacology and Clinical Aspects. Springfield, Illinois. Charles C Thomas, publisher.

76. Goodall, McC., and H. Alton. 1969. Dopamine (3,4-dihydroxyphenylethylamine) metabolism in severe thermal injury. J. Clin. Invest. 48: 1761.

77. Crampton, C. W. 1920. The gravity resisting ability of the circulation; its measurement and significance (Blood Ptosis). Amer. J. Med. Sci. 160: 721.

78. Wagner, Henry N., Jr. 1959. Orthostatic hypotension. Bull. Johns Hopkins Hosp. 105: 322.

79. Thomas, Juergen E., and A. Schirger. 1963. Neurologic manifestations in idiopathic orthostatic hypotension. Arch. Neurol. 8: 204. 